\title{
La crítica como ética del cuidado de sí ${ }^{1}$
}

\author{
Un estudio en torno a la Ilustración y las prácticas de sí \\ greco-latinas en Michel Foucault.
}

The critique as ethics of self-care A study around the illustration and the Greece-Latin
practices of the self in Michel Foucault

José Ignacio Scasserra ${ }^{2}$

Universidad de Buenos Aires, Argentina

Recepción: 9 de agosto del 2018

Evaluación: 15 de septiembre del 2018

Aceptación: 22 de octubre del 2018

1 Este artículo es resultado de una primera investigación realizada en torno a los trabajos de Michel Foucault de los años 80. Fue realizado en el marco del proyecto de investigación "La crítica de Michel Foucault como antecesora de la teoría butleriana de la performatividad" dependiente del Instituto de Estudios interdisciplinarios de Género de la Universidad de Buenos Aires, con financiación de la propia universidad.

2 Profesor de enseñanza superior y media en filosofía (Universidad de Buenos Aires). Actualmente se encuentra cursando la maestría Estudios Interdisciplinarios de la Subjetividad (UBA).

Correo electrónico:j_scasserra@hotmail.com 


\section{Resumen:}

El trabajo se propone evaluar el comentario de Michel Foucault al texto de Kant “QQué es la ilustración?” con el fin de dar cuenta de las continuidades que dicho comentario presenta para con la genealogía de las prácticas de sí greco - latinas que Foucault se encontraba estudiando entre los años 1980 y 1984, apartándose de este modo de una lectura que considere los trabajos foucaultianos sobre la Ilustración como un momento aislado de su producción en los años 80. Para ello, en primer lugar, se desarrollará el texto kantiano sobre la ilustración, luego abordaremos el modo en que Foucault enmarcó sus trabajos en dichos años en el prólogo de El uso de los placeres, para finalmente dirigirnos a los dos textos de Foucault sobre la ilustración. Nuestro desarrollo será realizado prestando atención a un triple gesto que creemos encontrar tanto en el texto kantiano como en el foucaultiano: la descripción, la adscripción, y la prescripción de ambos autores hacia el proyecto ilustrado. Dicha "similitud estructural” nos permitirá ordenar los desarrollos de ambos.

Palabras clave: Ilustración, ética, identidad, historia, Michel Foucault.

\section{Abstract:}

The work proposes to evaluate the commentary of Michel Foucault to the text of Kant "What is illustration?" with the goal of telling about the continuities that such commentary presents towards the genealogy of the Greece-Latin practices of the self that Foucault studied between 1980 and 1984, going ahead of a reading that considers the works of Foucault about the illustration as an isolated moment of his research in the 80s. To achieve this, in first place, the Kant work upon illustration will be developed and then, we will consider the manner in which Foucault enclosed his works in those years in the prologue found in The use of pleasures. Finally, we move towards two works of Foucault about the illustration. Our development will be done including a triple gesture that we may find in both the work of Kant as well as in the work of Foucault: the description, adscription and the prescription of both authors towards the illustrated project. Such "structural similarity" will allow us to organize their development.

Keywords: Illustration, ethics, identity, history, Michel Foucault. 


\section{La critique en tant qu'éthique du soin de soi : Une étude autour des}

\section{Lumières et des pratiques du moi gréco-latines chez Michel Foucault.}

\section{Résumé:}

Ce travail a pour objectif d'évaluer le commentaire de Michel Foucault à propos du texte de Kant "Qu'est-ce que les Lumières » afin de rendre compte des continuités que ce commentaire présente avec la généalogie des pratiques du moi gréco-latines que Foucault étudiait entre 1980 et 1984, s'éloignant ainsi d'une lecture qui considère les œuvres de Foucault sur les Lumières un moment isolé dans sa production des années 80 . Pour ce faire, nous développerons d'abord le texte kantien sur les Lumières, puis nous aborderons la façon dont Foucault a encadré ses œuvres de ces années-là dans le prologue de L'Utilisation des plaisirs, pour enfin nous tourner vers les deux textes de Foucault sur les Lumières. Notre développement se fera en prêtant attention à un triple geste que nous croyons retrouver dans le texte kantien comme dans le foucaldien: la description, l'attribution et la prescription des deux auteurs envers le projet illustré. Cette « similitude structurelle » nous permettra d'ordonner les développements des deux.

Mots-clés: illustration, éthique, identité, histoire, Michel Foucault.

\section{A crítica como ética do cuidado de si: um estudo em torno ao Iluminismo e as práticas de si greco-latinas em Michel Foucault}

\section{Resumo:}

Este trabalho propor avaliar o comentário de Michel Foucault sobre o texto de Kant "O que é o Iluminismo" com fins de compreender as continuidades que esse comentário apresenta para a genealogia das práticas de si greco-latinas que Foucault encontrava-se estudando entre os anos 1980 e 1984, afastandose desse modo uma leitura que considere os trabalhos foucaulteanos sobre o iluminismo como um momento isolado de sua produção nos anos 80 . Para isso, no primer momento, se desenvolverá o texto kantiano sobre o iluminismo, logo, abordaremos o modo em que Foucault molda seus trabalhos em esses anos nos prologo do Uso dos prazeres, para finalmente, nos dirigir aos dois textos de Foucault sobre o iluminismo. Nosso desenvolvimento é feito com atenção a um triplo gesto que acreditamos encontrar tanto no texto kantiano como no foucaulteano: a descrição, a adstrição e a prescrição de ambos autores para o projeto ilustrado. Essa "similitude estrutural" permitirá ordenar os desenvolvimentos de ambos.

Palavras-chave: Iluminismo, ética, identidade, história, Michel Foucault. 


\section{Introducción General}

"Para Foucault, preguntarse por la Ilustración equivalía también a preguntarse ¿Quiénes somos? Una vez más, este interrogante no puede resolverse, en su caso, profundizando en una naturaleza humana transhistórica poseedora de verdades esenciales. ¿Quiénes somos? no es equivalente a ¿Qué es el hombre? El problema que se plantea es más bien el de un modo de ser siempre singular e histórico. Por esta razón, puede decirse que ¿Quiénes somos? constituye una pregunta crítica: remite, hacia atrás, a una serie de investigaciones históricas orientadas a recorrer la genealogía de nuestras identidades, a poder pensarlas en una precariedad histórica esencial, y hacia adelante, a una transformación ética de nosotros mismos, a la invención política de nuevas subjetividades. ¿Quiénes somos? significa, a la vez, ¿de qué sintesis históricas está constituida nuestra identidad? Y ¿cómo podríamos ser de otro modo?

(Grós, 2007, p. 127)

Los trabajos de Michel Foucault, sensibles de recibir múltiples interpretaciones e hipótesis de lectura, han sido objeto en los últimos años de un atento relevamiento que ha buscado concentrarse en sus trabajos sobre la ética y el sujeto ${ }^{3}$. La producción foucaultiana de los años 80, que concluye con su muerte en 1984, se propone realizar un estudio de las formas de subjetivación que se concentra, en primera instancia, en las prácticas de cuidado de sí greco - latinas, para luego poner el acento en los vínculos que dichas prácticas establecen para con las formas aletúrgicas ${ }^{4}$, es decir, de producción de verdad ${ }^{5}$. Este buceo arqueológico-genealógico, que sorprendentemente se dirigió a épocas más extrañas que el común de la producción foucaultiana

3 Al respecto, destacamos: Alvarez Yágüez (2013); Gros, (2010); Gros y Levy, (2004): Castro, (2008); Díaz Marsá, M., (2014). Asimismo, creemos encontrar lecturas más creativas y apropiadoras en Butler, (2005) y Hekman, (2010).

4 En palabras de Foucault, las formas de aleturgia quedan definidas en Del gobierno de los vivos como el "conjunto de los procedimientos posibles, verbales o no, por los cuales se saca a la luz lo que se postula como verdadero en oposición a lo falso, lo oculto, lo indecible, lo imprevisible, el olvido, y decir que no hay ejercicio del poder sin algo parecido a una aleturgia" (Foucault, 2014, p. 24)

5 Cabe aclarar, siguiendo a Marcelo Raffin, que "Foucault se ocupó del problema de la verdad a lo largo de toda su obra, desde las primeras investigaciones, y que este no desaparece a lo largo de la década de 1970" (Raffin, 2016, 130). Tener en cuenta este recorrido nos aleja de interpretar que el autor "descubre" el problema de la verdad en determinado momento; por el contrario, entendemos que las variaciones observadas dentro de sus trabajos se deben meramente a un cambio de foco desde donde abordar problemáticas similares. 
(que solía anclar sus análisis en la modernidad), tenía sin embargo intenciones muy similares a las que habrían animado todas sus investigaciones: se trataba de dar cuenta de las formas de veridicción y de gubernamentabilidad ${ }^{6}$ que nos constituyen como aquello que somos: una pregunta por la producción de la propia identidad, como bien señala Grós en la cita escogida como encabezado, que intenta buscar respuestas en la historia de ciertos problemas comunes (Gros, 2007, p. 127).

En medio de sus trabajos focalizados en Grecia y Roma, el autor retornaría, sin embargo, a la modernidad. A lo largo de su primera clase del curso de 1983, El gobierno de sí y de los otros, y en sus dos textos de título "¿Qué es la Ilustración?" (el primero, del mismo año, y el segundo, de 1984) el autor abordaría el problema del legado moderno sobre nosotros bajo la problemática de la Ilustración, realizando una recepción y apropiación del célebre texto de Inmanuel Kant sobre las luces en donde buscará reelaborar sus lineamientos bajo nuevas claves de lectura ${ }^{7}$. La problemática por la propia identidad, como veremos más adelante, encontrará allí una doble valencia de saberse históricamente producida, pero éticamente disputable.

Ante este enfoque sobre la producción histórica de la identidad y del sujeto, la propuesta de este trabajo será reponer, desarrollar y evaluar el comentario foucaultiano al texto kantiano, con el fin de destacar sus vínculos con los trabajos sobre las prácticas de cuidado de sí greco-latinas ${ }^{8}$. De este modo buscamos alejarnos de una lectura que comprenda el rodeo por la Ilustración como un gesto aislado de este "último Foucault"; por el contrario, creemos que la recepción del texto kantiano se encuentra en absoluta consonancia con lo que el autor se encontraba investigando en esa época. Para realizar

6 Utilizamos las expresiones "veridicción” y "gubernamentabilidad” para señalar el desplazamiento metodológico que resalta el propio Foucault en el período que nos proponemos abordar. Al respecto, el autor dirá, en la primera clase de El gobierno de sí y de los otross, que se ha propuesto "sustituir la historia de los conocimientos por el análisis histórico de las formas de veridicción; sustituir la historia de las dominaciones por el análisis histórico de los procedimientos de la gubernamentalidad, y sustituir la teoría del sujeto o la historia de la subjetividad por le análisis histórico de la pragmática de sí y las formas adoptadas por ella" (Foucault, 2010, p. 21).

7 No desconocemos que dicha problemática ya había sido abordada por Foucault en su conferencia de 1978 “¿Qué es la crítica?”. La excluimos de nuestra enumeración previa por no constituir un "regreso" a la modernidad en medio del tratamiento de las prácticas de sí greco-latinas a lo largo de los años 80, movimiento que deseamos abordar en el presente artículo.

8 Es importante destacar que nos concentraremos en el trabajo arqueológico-genealógico del autor en su Historia de la sexualidad II: El uso de los placeres, y no en sus estudios sobre las formas aletúrgicas, que buscaremos abordar en investigaciones ulteriores. 
dicha vinculación, en primer lugar, desarrollaremos una primera lectura de “¿Qué es la Ilustración?” de Kant, con el fin de destacar los elementos que juzguemos necesarios para comprender la lectura foucaultiana. Luego, nos concentraremos en los trabajos de Foucault, bosquejando primero un esquema general de la línea de trabajo del autor en el período que nos proponemos estudiar, con el fin de señalar los puntos que consideremos importantes para trazar los vínculos con los trabajos del autor sobre la Ilustración. En tercer lugar, abordaremos los textos foucaultianos de 1983 y 1984, con el mismo nombre que el texto kantiano. A lo largo de este recorrido, buscaremos sistematizar los textos sobre la Ilustración (entiéndase, el de Kant y el de Foucault) a partir de encontrar en ambos lo que llamaremos una "similitud estructural" que repite un triple gesto por parte de los autores de adscripción, descripción y prescripción para con el proyecto ilustrado. Dicha sistematización será subsidiaria de nuestro objetivo general: creemos que destacar estos tres puntos en ambos autores pondrá de relieve tres focos problemáticos que vinculan directamente a la crítica con la ética del cuidado de sí, desarrollo que manifestaremos en nuestra conclusión.

\section{La Aufklärung en 17849}

\section{Introducción}

En el año 1784, el periódico alemán Berlinische Monatschrift (Boletín mensual de Berlín) publicó respuestas de diversos autores en torno a una pregunta común: ¿Qué es la Ilustración? La desarrollada por Kant es la que dos siglos más tarde sería recepcionada por Michel Foucault. A continuación, buscaremos reponerla prestando especial atención a los elementos que servirán a Foucault para realizar su lectura de la Ilustración.

\section{La Aufklärung según Kant}

En su texto “QQué es la Ilustración?” Kant busca definirla como la salida del hombre de su minoría de edad, de la cual él mismo es culpable. Dicha minoría de edad no es comprendida como un rasgo etario, ni un defecto del entendimiento, sino que es meramente la incapacidad de los hombres de servirse de su propio entendimiento por ceder a la pereza y la cobardía,

\footnotetext{
9 La siguiente reposición es una revisión y ampliación de la que realicé en la ponencia "Dos proyectos Críticos" en el marco del II Coloquio de Gubernamentabilidad y Biopolítica, publicada en las actas del congreso (Rudriejo y Venier, 2018, p. 183 - 196)
} 
delegando en un libro, en un director de consciencia o en un médico la prescripción de sus pensamientos, su moral y sus prácticas respectivamente. Por el contrario, tener el valor de servirse del propio entendimiento, sin la conducción de ninguna autoridad, será el lema de la Ilustración.

La minoría de edad aparece como una comodidad, un vicio de hombres perezosos: será más sencillo permitir que un libro, un director de consciencia o un médico dictaminen el actuar y el pensar de uno mismo. Salir de dicho estado será asimilado por el autor al proceso de aprender a caminar, en el que algunas caídas y golpes parecen ser inevitables en vistas de lograr desplazarse libremente. Estas dificultades hacen que el hombre en su minoría de edad le cobre afecto a su estado, y hasta casi haga de él su propia naturaleza. Complementariamente, aquéllos que tutelan sus prácticas buscan preservarlo en ese estado por medio de "estatutos y fórmulas, instrumentos mecánicos de un uso, o mejor abuso racional de sus dotes naturales" con los que preservan "los grilletes de una perdurable minoría de edad" (Kant, 1999, p. 37).

Contra esto, el autor sostiene que pensar por uno mismo será una vocación de todo hombre. El autor parece comprender los conflictos políticos que su propio planteo implica: su apología al librepensamiento deberá, necesariamente, entrar en conflicto con la legitimidad de la obediencia política, problemática filosófica de central importancia a lo largo de la modernidad ${ }^{10}$. Por ello buscará traducir sus consideraciones a términos lo más inofensivos posibles (Denker, 1974, p.10): será necesario trazar los límites de la actitud ilustrada para con respecto al mandato y la obediencia. Por ello, se abriría una brecha entre el uso privado y el uso público de la razón, los cuáles presentan fuertes diferencias al modo en que lo comprendemos hoy en día. Dentro del uso privado de la razón, el hombre es un mero funcionario que debe obedecer las órdenes de sus superiores sin posibilidad de abrir polémicas ni discusiones, para mejor funcionamiento de la maquinaria social, encontrando así el uso de su razón severamente limitado. Ahora bien, en su uso público, la razón será

10 En este punto creemos importante comprender el planteo kantiano en un marco discursivo más general donde encuentra interlocutores como Thomas Hobbes, John Locke o Jean Jaques Rousseau. En efecto, el principal esfuerzo de la tradición contractualista es el de legitimar la obediencia política de los súbditos para con respecto al soberano, tarea que se muestra urgente una vez que han aceptado que las distinciones y la sumisión bajo la convivencia social son artificiales y secundarias frente a la libertad e igualdad del hombre antes de su pertenencia a un cuerpo político (Dotti, 1994, p. 57) Creemos que en este punto Kant no se muestra ni inocente ni desconocedor de dichas problemáticas. 
ejercida por un docto ante la totalidad de sus lectores, mediante un uso libre y capaz de poder señalar falencias o discrepancias que presente para con los modos de gobierno. Dicha división permitirá el ejercicio del libre pensamiento mientras mantiene el respeto y la existencia de las leyes. Asimismo, queda garantizando de este modo la racionalidad de la legislación, ya que la misma se verá sometida a minuciosos análisis por parte del uso público de la razón, que se verá habilitado a señalar sus problemas, siempre y cuando busque el bien de la república.

Obediencia y autonomía se ven compatibilizadas en virtud de una figura invocada por Kant: el señor del mundo (entendamos, la razón ${ }^{11}$ ) que dictamina "razonad tanto como queráis y sobre lo que queráis; pero obedeced!" (Kant: 1999, 39). Si las leyes se someten a los límites de la razón, no habría modo en que un pueblo ilustrado desee transgredirlas. La autonomía liberal resuena en lo profundo del texto: "La piedra de toque de todo aquello que pueda decidirse como ley de un pueblo reside en la pregunta: ¿podría imponerse un pueblo a sí mismo semejante ley? (Kant, 1999, p. 41).

Para concluir, la actualidad de Kant será elaborada en términos de "época de ilustración", aún no ilustrada. Es entendida como un proceso, no un punto de culminación. Allí, el autor ejercerá su propio uso de razón pública al realizar una apología al gobierno de Federico II, rey de Prusia: encontrará allí un rey ilustrado que permite el ejercicio público de la razón, y atiende a la autonomía de la misma para la sanción de sus leyes. Dicha actitud ilustrada por parte de Federico II es destacada por Kant especialmente en materia de libertad religiosa: en efecto, todo el texto coloca el acento de la ilustración en los usos de la racionalidad pública con respecto a la religión. Esto se debe a que, según Kant, con respecto a artes y ciencia, no se observa tanta tendencia por parte de los señores a generar tutela. Sin embargo, la Aufklärung no se agota allí: un verdadero proyecto político ilustrado no teme en permitir que sus súbditos hagan uso público de la razón con respecto a la legislación en general. Recoge allí Kant una paradoja del súbdito a gobernar: "Un grado mayor de libertad civil parece favorable a la libertad del espíritu del pueblo y también le fija límites infranqueables; un grado menor le proporciona, por el contrario, el espacio en que extenderse según todas sus facultades" (Kant,

11 Sin embargo, en El Gobierno de sí y de los otros Foucault interrogará sobre quién es este "señor del mundo". Sus candidatos serán Dios, la razón, o Federico II. Según el autor, no se trata del primero, se trata un poco del segundo, y principalmente es el tercero (Foucault, 2010, p. 51). 
1999: p. 42). Mientras los límites artificiales siempre pueden ser sorteados, el límite infranqueable de la razón (aquél postulado por la obra crítica de Kant, que no desarrollaremos aquí) ${ }^{12}$ se impondrá al permitir su uso público por parte de los súbditos, y eso será lo que permitirá tratarlos conforme a la dignidad que merecen.

\section{Conclusión}

Recojamos de la reposición previamente realizada un triple gesto kantiano de adscripción, prescripción y descripción para con respecto a la Ilustración. Encontramos un interés de Kant, en primer lugar. por adscribir al proyecto ilustrado: la Aufklärung es algo que sucede en su actualidad y de la cual, como escritor, se sabe parte. En segundo lugar, Kant intentará describir la Ilustración, ya que da cuenta de los elementos principales que conforman dicho proceso (salida de minoría de edad, uso autónomo de la razón). Finalmente, el autor buscará prescribir un programa para la Aufklärung: sólo podremos hablar de época ilustrada si se respeta la doble vía racional que armoniza el ejercicio del librepensador con la obediencia política necesaria para el funcionamiento de la maquinaria social, es decir, la distinción entre uso público y privado de la razón.

\section{El programa foucaultiano de los años 80}

\section{Introducción}

A continuación, nos proponemos dar cuenta de los lineamientos generales de la producción foucaultiana de los años 80, con el fin de poder enmarcar la apropiación que el autor realizó en dichos años del texto "¿Qué es la ilustración?” de Kant. La producción de Michel Foucault, incluso para un período tan breve como el que nos proponemos recortar, resulta vasta e inabarcable para los fines de este artículo; por ello, a fines de ceñirnos a nuestro objetivo, nos limitaremos al desarrollo que el propio autor realizó en el prólogo de su libro de 1984, El uso de los placeres, nutriendo nuestro esquema ocasionalmente con contribuciones de los cursos dictados por el autor en dichos años.

$12 \mathrm{Al}$ respecto, es importante resaltar las continuidades entre el proyecto Ilustrado de Kant y su proyecto crítico. En efecto, los límites del propio entendimiento que el sujeto de la crítica deberá respetar son garantes de que el ciudadano ilustrado no traspase las limitaciones de su uso público de la razón. Recuperé dicho problema en "Dos proyectos Críticos", expuesto en el II Coloquio de Gubernamentabilidad y Biopolitica (Rudriejo y Venier, 2018, p. 183 - 196). 


\section{Una historia ética de la sexualidad}

El autor comienza su reconocido libro dando cuenta de cuál había sido su objeto de interés: se trataba de pensar la sexualidad, término relativamente reciente (aparecido a principios del siglo XIX), buscando tomar distancia del mismo y analizando los contextos a los que se habría visto vinculado. La sexualidad será entendida por el autor como un "foco de experiencia", es decir, como "la correlación, dentro de una cultura, entre campos de saber, tipos de normatividad y formas de subjetividad" (Foucault, 2010, p. 10). Para realizar una comprensión cabal del término, se debía prestar atención a las formaciones de saber desde una perspectiva arqueológica, esto es, dando cuenta de las condiciones de emergencia de los saberes con respecto a la sexualidad y no comprendiendo su historia como un progreso del conocimiento hacia la objetividad. El enfoque genealógico se concentraría en los tipos de normatividad que rodearían a la sexualidad; el mismo no trataba de hacer una historia de los diversos conceptos de deseo, "sino más bien de analizar las prácticas mediante las cuáles los individuos se vieron llevados a prestarse atención a ellos mismos, a descubrirse, a reconocerse y declararse como sujetos de deseo" (Foucault, 2010, p. 11) ${ }^{13}$.

Trazar esta historia lleva al autor a operar un desplazamiento sobre su enfoque, ya no concentrándose primordialmente ni en el saber, ni en el poder, sino incorporando dichos ejes para concentrarse en aquello que se ha llamado "sujeto": "convenía buscar cuáles son las formas y las modalidades de la relación consigo mismo por las que el individuo se constituye y se reconoce como sujeto" (Foucault, 2010, p. 12). Fue en esa historia de la relación del individuo consigo mismo, en la cual el sujeto es comprendido como resultado y no como punto de partida, donde el autor encontró la necesidad de rastrear dicha problematización hacia la antigüedad greco-latina. Las preguntas lo guiaron hacia una fuerte inquietud ética en torno a la sexualidad, el placer y sus actividades, que orbitaban no solamente en torno al gobierno de uno mismo, sino por el gobierno de los otros.

Pero el proyecto de Foucault, si bien encuentra un ancla circunstancial en la sexualidad, no se agota allí. El autor habría dado, en la antigua Grecia,

13 Como señalamos en la nota al pie 6, no ignoramos que este triple enfoque sería, en esos años, reconfigurado por Foucault en términos de "formas de veridicción", "de gubernamentabilidad" y de "pragmática de sí". Sin embargo, con el fin de atenernos a los términos que el autor usa en el texto que nos encontramos reponiendo, mantenemos las expresiones "saber", "poder" y "sujeto". 
con un conjunto de práctica llamadas "artes de la existencia", cuyo foco no está puesto en las reglas que dirigen la conducta, sino en los modos en que los individuos buscan transformarse a sí mismos y "hacer de su vida una obra que presente ciertos valores estéticos y responda a ciertos criterios de estilo" (Foucault, 2010, p. 17). Estas prácticas de sí, si bien poseen una fuerte ancla en la sexualidad, no se agotan allí e incorporan regímenes dietéticos, procederes económicos, e incluso, más adelante, una íntima y profunda relación del individuo para con los discursos de verdad. Allí la constitución de uno mismo como sujeto hablante, interventor en el ámbito político, hombre adulto y libre, se dibuja como el foco de las inquietudes, por lo que debemos prevenirnos de leer en estas investigaciones un "retorno salvador" (Grós, 2007, p. 121) del sujeto, o un "regreso al subjetivismo" (Scasserra, 2017); por el contrario, se trata de una relación ética de uno con uno mismo en el cuál toda la experiencia histórica con los otros se pone en juego.

Finalmente, Foucault reconoce que con la emergencia del cristianismo estas prácticas perdieron su autonomía y fueron gradualmente desapareciendo en el marco del poder pastoral y las dimensiones institucionales de la educación, la medicina o la psicología. Sin embargo, no por ello admite su desaparición: menciona, en una nota al pie (Foucault, 2010, p. 17) el trabajo de Walter Benjamín sobre Baudelaire ${ }^{14}$ como un ejemplo de revitalización moderna de dichas prácticas de sí.

\section{Conclusión}

De la esquemática y breve reposición previa recojamos un enfoque que buscó poner el acento no en un sujeto constituido por tramas de saber y poder que determinarían de lleno sus modos de existir, sino en un modo más complejo que abre diversos frentes problemáticos a partir de su constitución moral y ética. Dicha conformación, lo sabemos, está signada por las determinaciones discursivas y gubernamentales epocales, pero no se agota necesariamente allí: si la moral será entendida como la relación (nunca unidireccional y siempre esquiva) del individuo para con determinado código prescriptivo, la ética será comprendida de ahora y en adelante por el autor como el ámbito de disputa que abre el individuo en su relación consigo mismo.

14 Se trata de un conjunto de artículos que pueden encontrarse recopilados bajo el título Baudelaire (Benjamin, 2014). 


\section{La Ilustración según Michel Foucault.}

\section{Introducción}

Como se puede ver a partir del apartado anterior, los textos sobre la ilustración de 1983 y 1984 presentan, en alguna medida, una excepción con respecto a la genealogía en la que el autor se encontraba trabajando en esos años. La estética de la existencia, el cuidado de sí, la ascética de los primeros tiempos cristianos, pueblan sus textos y cursos bosquejando un arco de la historia de la filosofía que llega hasta nuestros días. Ahora bien, en medio de dichos desarrollos (y por primera vez en éste período, en la primera clase de $1983^{15}$ ) el nombre de Kant y la Ilustración ocupan un lugar central. A continuación, repondremos el modo en que Foucault se apropia del texto kantiano con una lectura sistemática que proponga (principalmente, sobre el texto de 1984) tres gestos apropiativos por parte del filósofo francés que emulen aquéllos señalados en el texto de Kant. Si en el texto del filósofo de Konigsberg encontramos un triple gesto de adscripción, descripción y prescripción hacia la Ilustración, creemos que es posible rastrear un gesto análogo por parte de Foucault, pero en términos de reescritura de la crítica como histórica, actitudinal y transgresora, respectivamente.

\section{La ilustración en 1983.}

Lo primero que observará el autor es que el texto kantiano introduce una novedad con respecto a las problemáticas filosóficas de su tiempo: el problema de la actualidad. La pregunta por el acontecimiento, el “¿Qué pasa hoy?” se encontrará en la centralidad del texto. Dicha actualidad será comprendida no como una sucesión de eventos que el filósofo contempla desde una exterioridad, para garantizar su objetividad o neutralidad, sino teniendo en cuenta que se trata de mostrar "cómo quien habla en tanto pensador, en tanto científico, en tanto filósofo, forma parte él mismo de ese proceso, y (...) cómo tiene que desempeñar un rol determinado en ese proceso, del cual por lo tanto se sentirá a la vez elemento y actor". (Foucault, 2002, p. 67). La respuesta kantiana comprendería al filósofo y el pensador en tanto elemento de un

15 Si bien es cierto que Foucault ya había tocado el problema de la crítica y la ilustración en su conferencia “¿Qué es la Crítica?” (1978), la excluimos por ahora de nuestras consideraciones por enmarcarse más en sus trabajos biopolíticos sobre la modernidad que en sus investigaciones sobre la constitución subjetiva. 
proceso y actor del mismo. Esto será posible solamente en virtud de que la Ilustración fue un movimiento autoconsciente: se dio su propio nombre, su propia situación con respecto al pasado, al porvenir y a su tarea para con el presente, es decir, la de promover las luces del saber sobre la oscuridad de la ignorancia (con las consecuencias políticas y religiosas que implicaba).

Pero dicha consciencia de ser parte de un momento específico no se agota en ser meramente un eslabón en una cadena del pensamiento: Foucault podrá leer allí la "pertenencia a cierto nosotros, a un nosotros que se remite a un conjunto cultural característico de su propia actualidad" (Foucault, 2002, p. 68). Ese nosotros actual, en tiempos de Kant se vio demarcado de dos modos: la ilustración misma, y la "voluntad de revolución" con respecto al proceso francés. Sobre esta última, se la toma como un acontecimiento el cuál, sin importar su éxito y su fracaso, opera de índice para con respecto al progreso humano y a su disposición moral, en la medida en que podía percibirse, en líneas generales, un entusiasmo compartido por aquéllos que no habrían participado de dicho proceso.

A partir del desarrollo de este "pensamiento de la actualidad", el texto propone una escisión de planteos dentro y a partir de la filosofía kantiana. En efecto, Foucault lee dos vertientes críticas: por un lado, la que llamará una "analítica de la verdad" la cual interrogará por los límites y alcances del conocimiento verdadero, y una analítica del presente, o una "ontología de nosotros mismos", la cual interrogará por la actualidad y el campo actual de experiencias posibles. Allí pues, la filosofía contemporánea habría heredado una decisión: nos encontramos ante la disyuntiva de optar por una "filosofía crítica que se presentará como una filosofía analítica de la verdad en general, o bien se puede optar por un pensamiento crítico que tomará la forma de una ontología de nosotros mismos, de una ontología de la actualidad" (Foucault, 2002, p. 80). En palabras de Virginia Cano, Foucault habría realizado una "lectura bifronte" (Cano, 2013, p. 243) de la obra kantiana, y a partir de dicha división habría planteado dos escenarios problemáticos heredados por la filosofía contemporánea, optando él mismo por inscribirse en el segundo de los mismos ${ }^{16}$.

16 Es importante remarcar que, de cualquier forma, estas "dos vertientes" que tan divididas aparecen en este momento del texto, en otras ocasiones serán puestas en vinculación por el propio Foucault. $\mathrm{Al}$ respecto, ver la primera clase del curso El gobierno de sí y de los otros, más específicamente, el apartado titulado "La sombra de las tres críticas", (Foucault; 2010, p. 46 - 48) 
Recuperemos de estos desarrollos la primera filiación que podemos realizar para con respecto a la problemática que abrió nuestro texto y que creímos encontrar en el período escogido de la obra foucaultiana para nuestro estudio: en efecto, el problema del presente y de la actualidad debe ser aprehendido en clave de una pregunta por la propia identidad en su constitución histórica, y no bajo el modo de respuestas universales que busquen hipostasiar un concepto de "lo humano" determinado. Veremos a continuación cómo en esta pregunta por el "¿quiénes somos?”, se pondrá en juego la relación del individuo consigo mismo para disputar su propia constitución como sujeto ético.

\section{La Ilustración en 1984}

\section{Observaciones iniciales}

Un año después, la pregunta sobre la ilustración insiste y persiste: fue arrojada hace dos siglos al público de lectores imprudentemente, y hace dos siglos que la filosofía no consigue darle una respuesta, aunque sin lograr desembarazarse de la cuestión. Al respecto, Foucault realiza tres observaciones a comienzos de su texto:

1) La pregunta fue respondida por Moses Mendelssohn en el mismo diario dos meses antes que Kant; sin embargo, éste no había leído la respuesta de aquél a la hora de escribir su propio texto. Se inaugura allí la posibilidad de pensar una "ilustración judía” y una "ilustración cristiana”, las cuáles emparentarían ambos mundos en un proceso común: lo alemán y lo judío reconocen una raíz que los emparenta y ata sus destinos ${ }^{17}$.

2) A la interioridad de la tradición cristiana, el texto es sin embargo una novedad en su modo de comprender el presente: este ya no será la pertenencia a determinada época del mundo (gesto que Foucault identifica con la filosofía platónica), ni un signo que anuncia el futuro (propio del pensamiento agustiniano), ni como la transición hacia la aurora de un nuevo mundo (esfuerzo que se puede leer en Vico). En el caso de Kant, el presente será definido de forma íntegramente negativa: será una salida, un resultado. No es una totalidad ni una consumación, sino que marca su diferencia con respecto al ayer en la forma de la privación.

$17 \mathrm{Al}$ respecto, Foucault se expendería más sobre el tema en su primera clase de El gobierno de sí y de los otros (más específicamente, pgs. 25 - 28) 
3) Dicha salida es un proceso en desarrollo que se presenta como una tarea y una obligación. Esta obligación jugaría de espejo con la propia responsabilidad que el hombre tiene de su minoría: allí pues, "hay que concebir que no podrá salir de él sino mediante un cambio que él mismo operará sobre sí mismo" (Foucault, 2002, p. 85). La sustitución que el individuo hace de su intelecto por las autoridades mencionadas por Kant (libro, director de consciencia y médico) serán la causa de la minoría de edad, ya que harían de mediadores en la relación de uno consigo mismo; por ende, es obligación de cada individuo ejercer la razón en su uso autónomo para ingresar en la "mayoría de edad".

Ante nosotros queda un tipo de interrogación filosófica que problematiza nuestra relación con el presente, nuestro modo de ser histórico, y la constitución de nosotros mismos como sujetos autónomos. En términos de Álvarez Yagüez,

el tiempo penetra el pensamiento mismo, determina su tarea, le da una finalidad (...) le confiere una naturaleza distinta puesto que en su interior lo más propiamente universal, el estricto plano del pensar, del concepto, de la teoría, de lo universal, se ve cruzado por la temporalidad, lo concreto, lo particular (Álvarez Yagüez, 2015, p. 20)

En efecto, pareciera que Foucault, recuperando elementos del texto de 1983, insiste en una primera ruptura con respecto a Kant que no queremos dejar de señalar: al relacionar historia y crítica, mediante un trabajo paciente de rastreo de los propios límites históricos, la crítica ya no será de condiciones trascendentales y universales, sino de las particularidades y sus discontinuidades. Foucault habría decidido, en efecto, por la segunda de las dos vertientes que señaló en su texto de 1983 por medio de su "lectura bifronte".

\section{Su caracterización negativa}

Nuestra ligazón con dicho proceso no se debe a repeticiones de doctrina, sino simplemente a la "reactivación permanente de una actitud; es decir, un ethos filosófico que podría caracterizar como crítica permanente de nuestro ser histórico" (Foucault, 2002, p. 97). Este ethos será desarrollado por el autor, en primer lugar, negativamente de dos maneras:

1) Se propone rechazar el llamado "chantaje" de la ilustración, es decir, la alternativa impuesta de forma simplista y autoritaria que busca 
convencer a la actividad filosófica de que es necesario pronunciarse a favor o en contra de aquélla. Para sortear dicha disyunción, el autor intentará comprendernos como seres históricamente determinados por la Ilustración, pero con la tarea de realizar investigaciones históricas que apunten hacia los límites de lo actual: es decir, "hacia lo que no es o ya no es indispensable para la constitución de nosotros mismos como sujetos autónomos" (Foucault, 2002, p. 98).

2) Otro camino que debe ser evitado es el de confundir a la Aufklärung con el humanismo. Mientras el autor lee en éste último un conglomerado de temas ligados a juicios de valor que justifican concepciones del hombre a las que está obligadas a recurrir, el pensamiento crítico del presente implica una creación permanente de nosotros mismos en nuestra autonomía. Aquí, el pensamiento histórico de la identidad se encuentra nuevamente operando: como al inicio de nuestro texto, en la cita de Fréderic Grós, la pregunta por el "¿quiénes somos?” no se asimila a la pregunta por "¿Qué es el hombre?". Con este nuevo movimiento, Foucault le ha usurpado de las manos la crítica a Kant, dislocando la pregunta antropológica (que era en efecto la cuarta pregunta kantiana) y reconfigurándola en términos históricos.

De esta caracterización negativa, queremos destacar un segundo gesto apropiativo: la reescritura de la crítica en términos actitudinales introduce en ella novedades con respecto a la constitución subjetiva que será (a partir de la primera ruptura) siempre histórica y dependiente de un trabajo, una creación permanente de nosotros mismos en nuestra autonomía, y nunca una interrogación por las condiciones universales y antropológicas del sujeto. Asimismo, es importante destacar que la constitución subjetiva se verá permeada por el presente al cuál pertenecemos, por lo que no creemos posible leer allí una primacía del sujeto en tanto instancia fundacional.

\section{Su caracterización positiva}

En segundo lugar, el ethos revitalizado por la aufklarüng es caracterizado de forma positiva por medio de proponer una ontología histórica de nosotros mismos.

1) En tanto "actitud límite", que busque resaltar las fronteras y las zonas liminares, rechazando dualismos que creen rápidamente "afueras" y "adentros" con pretensiones excluyentes. Recuerda ahí Foucault: para 
la crítica kantiana, se trataba de rastrear los límites y alcances para subsumirnos a ellos; ahora bien, allí donde Kant proponía respetar los límites dados a priori, Foucault nos instará a rastrear los modos en que éstos pueden ser tensionados. "Se trata en suma de transformar la crítica ejercida en la forma de la limitación necesaria en una crítica práctica en la forma del franqueamiento posible" (Foucault, 2002, p. 102). Esta inversión tendrá consecuencias inmediatas: la crítica ya no debe buscar estructuras universales ni formales, sino que deberá adoptar la forma de una investigación histórica a través de los acontecimientos que nos hicieron constituirnos como sujetos. Es en este punto que el autor enlaza sus dos columnas metodológicas dentro del proyecto crítico: en lugar de ser trascendental, la crítica será genealógica en su finalidad y arqueológica en su método. No tendrá como objetivo rastrear estructuras universales, sino los discursos que articulan nuestro pensamiento y nuestros eventos históricos, buscando deducir, ya no lo que nos es imposible hacer, sino "de la contingencia que nos ha hecho ser lo que somos, la posibilidad de no seguir siendo, pensando o haciendo lo que somos, hacemos o pensamos" (Foucault, 2002, p. 102).

2) En segundo lugar, el autor introducirá una dimensión experimental en la actitud histórico crítica, en vistas de que ésta no sea solamente un programa prescriptivo. Por ello, el intento de nuevos modos de ser y pensar se muestra fundamental para el autor; recuperando nuevamente sus trabajos sobre la ética greco latina, Foucault pareciera sugerir el "trabajo de nosotros sobre nosotros mismos en tanto seres libres" (Foucault, 2002, pp. 103 - 104) como prueba de los límites que somos posibles de franquear.

3) Finalmente, Foucault lidiará con los peligros de dicho análisis parcial y experimental. ¿No se incurriría en el riesgo de dejarse determinar por estructuras de las cuáles aún no hemos tomado conocimiento? Sobre esta cuestión el autor admite que, en efecto, siempre será así. Difícilmente podamos dar cuenta de la totalidad de la historia que nos hace ser lo que somos. Pero eso no quiere decir que la propuesta no tenga un orden. La apuesta, la homogeneidad, y la sistematicidad del proyecto crítico garantizarían su método y rastrearían recurrencias históricas que, al menos en occidente, se repetirían hasta nuestros días, no por una universalidad metahistórica, sino por la recurrencia en los focos de problematización. 
Estos últimos planteos bosquejan un tercer gesto apropiativo por parte de Foucault que presenta rupturas y continuidades para con el pensamiento kantiano. Se trata de una "inversión axiológica" (Cano, 2013, p. 247) en la cual Foucault nos instará ya no a respetar los límites dados a priori por la razón, sino a transgredirlos por medio de un trabajo sobre nosotros mismos (segunda apropiación) en virtud de haber comprendido que ésos límites son históricos y contingentes (primera apropiación).

\section{Conclusión: Una "similitud estructural"}

El propósito de esta conclusión parcial es el de sistematizar los extensos desarrollos previos por medio de rastrear una "similitud estructural" entre el texto foucaultiano y el texto kantiano, con el fin de dirigirnos ulteriormente a atisbar los vínculos que encontramos entre la Ilustración y la ética del cuidado de sí. Si propusimos un gesto de adscripción, descripción y prescripción de Kant para con la ilustración, creemos que podemos encontrar mismo esquema en el caso de Foucault, en analogía con los tres gestos de apropiación que arriba mencionamos. En efecto, el autor adscribe (en su texto de 1983) a la ilustración como movimiento que le ha legado la problemática del presente y el nosotros del cual se sabe parte. Asimismo, busca describir a la ilustración, ciñéndose en ocasiones a los términos de Kant, pero agregándole coordenadas actitudinales novedosas. Finalmente, propone su programa prescriptivo para el pensamiento crítico, el cual deberá tener como horizonte la posibilidad de dejar de pensar, sentir y hacer lo que nos ha sido legado, para producir nuevos modos virtuales y efectivos de existencia.

\section{Conclusión General}

Escribe Edgardo Castro "La relación de Foucault con Kant es, al mismo tiempo, de ruptura y de continuidad. (...) La arqueología y la genealogía se oponen, desde una perspectiva nietzscheana, a la disposición kantiana, antropológica, del pensamiento moderno" (Castro, 2011, p. 216). Creemos que esta cita condensa velozmente los resultados de los desarrollos previos. En efecto, y a modo de balance, hemos logrado dar con una apropiación foucaultiana del proyecto kantiano que busca servirse de la filosofía del konigsberguense, al mismo tiempo que lo historiza e interviene algunos de sus postulados principales. En las tres rupturas (y continuidades) mencionadas más arriba creemos que es posible rastrear lineamientos que vinculan la apropiación creativa de Foucault sobre la Ilustración con sus trabajos sobre la ética y el sujeto. 
La primera ruptura y continuidad que hemos podido observar es aquélla mediante el cual el autor busca adscribir a la pregunta por el presente y nuestra identidad, pero solamente en virtud de traducir dicha identidad a términos históricos, abandonando la última pregunta kantiana por el hombre. En efecto, el problema del presente y de la actualidad debe ser aprehendido en clave de una pregunta por la propia identidad en su constitución histórica, y no bajo el modo de respuestas universales que busquen hipostasiar un concepto de "lo humano" determinado. Este primer postulado puede servir de marco explicativo para un proyecto que se proponga rastrear las particularidades históricas greco-latinas mediante las cuáles un individuo se ve bajo la necesidad de instituirse como sujeto; en efecto, la pregunta por los modos en que el individuo se reconoce como sujeto de las tramas de saber y poder que lo constituyen sólo adquiere relevancia y sentido en virtud de abandonar una imagen previa del "hombre" que neutraliza los marcos de existencia posible en virtud de una supuesta "naturaleza humana".

El segundo gesto de apropiación ha sido aquél mediante el cuál Foucault describe el proyecto ilustrado ya no un proceso solamente cultural e histórico, sino directamente "actitudinal", el cuál es descripto en la modernidad por Foucault por medio de la obra de Baudelaire. Este ejemplo no es menor, si recordamos que, en El uso de los placeres, el autor también habría indicado, en una nota al pie, que Baudelaire representa en efecto una revitalización de las prácticas de sí. Por ende, "actitud moderna" y cuidado de sí se ven involucrados en un mismo argumento histórico, esta vez por medio del pensamiento heroico e irónico del presente Baudelaireano que encuentra su cristalización en el dandismo y la estilización de sí.

Finalmente, encontramos un tercer momento apropiativo en el modo en que el autor se considera heredero de la problemática ilustrada de la salida de la minoría de edad, pero entendiendo dicha salida de forma diferente: ya no cómo el modo en que los individuos reconocen los límites universales y $a$ priori de la razón, sino bajo la prescripción de identificar nuestros límites históricos heredados con el fin de disputarlos, tensionarlos y transgredirlos. Dicha trasgresión, desde Kant mismo, es comprendida como una disputa ética: la salida de la minoría de edad es, en efecto, resultado de un trabajo que el propio que el individuo realiza sobre sí mismo. Pareciera que podemos encontrar atisbos de una ética del "cuidado de sí" en la ilustración, rastreando en Kant consideraciones que excederían la ética deontológica que se habría 
leído en sus obras principales ${ }^{18}$. La sustitución que el individuo hace de sí mismo por las autoridades mencionadas por Kant (libro, médico, director de consciencia) serán la causa de la minoría de edad, ya que harían de mediadores en la relación de uno consigo mismo. Disputar dicho espacio será el trabajo ético que nos permitiría conquistar la mayoría de edad ${ }^{19}$. En Foucault, la vinculación del cuidado de sí como "práctica de libertad" se muestra en sintonía con estas consideraciones, buscando actualizar una relación ética del individuo consigo mismo que, si bien depende de las tramas de saber y poder que lo han conformado, las exceden y desbordan.

En efecto, el pensamiento crítico, una vez que ha sido tamizado por Foucault, encuentra un modo de insertarse en la historia de la ética del cuidado de sí individual, donde lo que se pone en juego es nuestra relación con nuestras propias herencias, prejuicios, blasfemias, violencias, mártires o dioses. No desconocer dichas herencias, sino que afrontarlas con la paciencia del arqueólogo y el coraje crítico de la genealogía fue la apuesta que Michel Foucault buscó revitalizar y proponer. Allí se juega una atenta mirada hacia nosotros mismos en tanto herederos bastardos, disputando nuevos modos de ejercer la libertad.

\section{Referencias}

Álvarez Yagüez, J. (2015) "Introducción: Una ética del pensamiento" en Foucault, Michel, La ética del pensamiento. Para una crítica de lo que somos, (ed. de Jorge Álvarez Yagüez), Madrid, Biblioteca Nueva.

Benjamin, W. (2014) Baudelaire, Madrid, Abada editores.

Butler, J. (2005) Giving an Account of Oneself, New York, Fordham University Press.

Cano, V. (2013) “Dar cuenta de nosotr@s mism@s: el coraje de la crítica en $J$. Butler y M. Foucault" en Femenías, M. L, Cano, V., Torricella, P. (comp.), Judith Butler, su filosofía a debate, Buenos Aires, Editorial de la facultad de Filosofía y Letras, Universidad de Buenos Aires.

\footnotetext{
18 Fundamentación de la metafísica de las costumbres y Crítica de la Razón Práctica

19 Sobre intentos de leer en Kant una posible ética del cuidado de sí, recomiendo los trabajos de Daniel Omar Perez (Perez, 2004: p. 19 -21).
} 
Castro, E. (2011) Diccionario Foucault, Buenos Aires, ed. Siglo XXI.

Castro, R. (2008) Foucault y el cuidado de la libertad. Ética para un rostro de Arena, Santiago de Chile, LOM.

Denker, R. (1974) "La teoría kantiana de las tres vías que conducen a la paz universal o los designios de la naturaleza de la historia" en Gerresheim, Eduard (ed), Inmanuel Kant. 1724/1974. Kant como pensador politico, (p. 5 - 16) Inter Nationes, Bonn - Bad Godesberg.

Díaz Marsá, M. (2014), Modificaciones. Ontología crítica y antropología política en el pensamiento de Foucault, Madrid, Escolar y Mayo.

Dotti, J. (1994) "Pensamiento político moderno", en Olaso, Ezequiel de (ed.), Enciclopedia Iberoamericana de Filosofía VI: Del Renacimiento a la Ilustración, Madrid, ed. Trotta, p. 53-76.

Foucault, M. (1995) “¿Qué es la Crítica? (Crítica y Aufklarüng)” trad. Javier de la Higuera, en Daimon Revista de Filosofía, N. 11, pg. 5 - 25

Foucault, M. (1999) “Respuesta a la pregunta: ¿Qué es ilustración?” (Traducción de Javier Alcoriza y Antonio Lastra), en Kant, Immanuel, En defensa de la Ilustración, Barcelona, Alba Editorial. (Formato electrónico).

Foucault, M. (2002) ¿Qué es la Ilustración?, Córdoba, ed. Alción.

Foucault, M. (2010) El gobierno de sí y de los otros, Buenos Aires, Ed. Fondo de cultura económica.

Foucault, M. (2010) Foucault, el coraje de la verdad, Madrid, Arena libros.

Foucault, M. (2010) Historia de la sexualidad: El uso de los placeres, Buenos Aires, Siglo XXI.

Foucault, M. (2010) Historia de la sexualidad: La inquietud de sí, Buenos Aires, Siglo XXI.

Foucault, M. (2013) El último Foucault. Voluntad de verdad y subjetividad, Madrid, Biblioteca Nueva.

Foucault, M. (2014) Del gobierno de los vivos, Buenos Aires, Ed. Fondo de cultura económica. 
Gros, F \& Carlos Lévy, (2004) Foucault y la filosofía antigua, Buenos Aires, Nueva Visión.

Gros, F. (2007) Michel Foucault, Buenos Aires, ed. Amorrortu.

Hekman, S. (2010) Feminist interpretations of Michel Foucault, Pennsylvania State University Press.

Kant, I. (2009) Crítica de la Razón pura, Buenos Aires, ed. Colihue.

Perez, D. (2004) “A questão do sujeito entre Kant e Foucault”, en Anais - IV Semana de Filosofía: a inclusão do sujeito no discurso filosófico. (p. 11- 22) / Departamento de Filosofía da Universidade estadual do centro - oeste - Guarapuava: UNICENTRO, 2004.

Raffin, M. (2016) "Verdad, poder y sujeto en las lecturas de Edipo de Michel Foucault", en Castro Orellana, Rodrigo y Salinas Araya, Adán (eds.), La actualidad de Michel Foucualt, España, Escolar y Mayo ed.

Scasserra, J. (2017) "Consideraciones acerca de un sujeto moral" en Reflexiones Marginales, núm. 39, Buenos Aires. Ed. digital disponible en http://reflexionesmarginales.com/3.0/consideraciones-acerca-deun-sujeto-moral-intentando-poner-en-funcionamiento-la-obra-demichel-foucault/

Scasserra, J. (2018) "Dos proyectos críticos" en Rudriejo y Venier (comp.) Gubernamentalidad y biopolítica: contribuciones para una ontología del presente, (p. 183 - 195), Universidad Nacional de Salta, Sa 